\title{
Oscillation Behavior for a Class of Differential Equation with Fractional-Order Derivatives
}

\author{
Shouxian Xiang, ${ }^{1}$ Zhenlai Han,, ${ }^{1}$ Ping Zhao, ${ }^{2}$ and Ying Sun ${ }^{1}$ \\ ${ }^{1}$ School of Mathematical Sciences, University of Jinan, Jinan, Shandong 250022, China \\ ${ }^{2}$ School of Electrical Engineering, University of Jinan, Jinan, Shandong 250022, China \\ Correspondence should be addressed to Zhenlai Han; hanzhenlai@163.com
}

Received 26 April 2014; Accepted 21 July 2014; Published 6 August 2014

Academic Editor: Shurong Sun

Copyright (C) 2014 Shouxian Xiang et al. This is an open access article distributed under the Creative Commons Attribution License, which permits unrestricted use, distribution, and reproduction in any medium, provided the original work is properly cited.

By using a generalized Riccati transformation technique and an inequality, we establish some oscillation theorems for the fractional differential equation $\left[a(t)\left(p(t)+q(t)\left(D_{-}^{\alpha} x\right)(t)\right)^{\gamma}\right]^{\prime}-b(t) f\left(\int_{t}^{\infty}(s-t)^{-\alpha} x(s) d s\right)=0$, for $t \geqslant t_{0}>0$, where $D_{-}^{\alpha} x$ is the Liouville right-sided fractional derivative of order $\alpha \in(0,1)$ of $x$ and $\gamma$ is a quotient of odd positive integers. The results in this paper extend and improve the results given in the literatures (Chen, 2012).

\section{Introduction}

Differential equations with fractional-order derivatives have gained importance due to their various applications in science and engineering such as rheology, dynamical processes in self-similar and porous structures, heat conduction, control theory, electroanalytical chemistry, chemical physics, and economics; for example, see [1-7]. It is well recognized that fractional calculus leads to better results than classical calculus.

Many articles have investigated some aspects of differential equation with fractional-order derivatives, such as the existence and uniqueness for $p$-type fractional neutral differential equations, smoothness and stability of the solutions, and the methods for explicit and numerical solutions; for example, see [8-16]. However, to the best of the author's knowledge very little is known regarding the oscillatory behavior of differential equation with fractionalorder derivatives up to now except for [17-27].

Grace initiated the study of oscillatory theory of FDE, and he considered the equations of the form

$$
D_{a}^{q} x+f_{1}(t, x)=v(t)+f_{2}(t, x), \quad \lim _{t \rightarrow a^{+}} J_{a}^{1-q} x(t)=b_{1}
$$

where $D_{a}^{q}$ denotes the Riemann-Liouville differential operator of order $q$ with $0<q<1$. In fact, the IVP is equivalent to the Volteria fractional integral equation:

$$
\begin{aligned}
x(t)= & \frac{b_{1}(t-a)^{q-1}}{\Gamma(q)} \\
& +\frac{1}{\Gamma(q)} \int_{a}^{t}(t-s)^{q-1}\left[v(s)+f_{2}(s, x(s))\right. \\
& \left.-f_{1}(s, x(s))\right] d s .
\end{aligned}
$$

He made use of the conditions:

$$
\begin{aligned}
x f_{i}(t, x)>0 \quad(i=1,2), \quad x \neq 0, t \geqslant a, & \\
\left|f_{1}(t, x)\right| \geqslant p_{1}(t)|x|^{\beta}, \quad\left|f_{2}(t, x)\right| & \leqslant p_{2}(t)|x|^{\gamma}, \\
x & \neq 0, \quad t \geqslant a,
\end{aligned}
$$

where $p_{1}, p_{2} \in C\left([a, \infty), \mathbb{R}^{+}\right)$and $\beta, \gamma>0$ are real numbers. He talked over the four cases of $f_{2}=0 ; \beta>1$ and $\gamma=1$; $\beta=1$ and $\gamma<1 ; \beta>1$ and $\gamma<1$. Besides that, he replaced $0<q<1$ with $m-1<q<m$ and got some results on the same cases by using an inequality; refer to [17]. 
Chen studied the oscillation of the differential equation with fractional-order derivatives:

$$
\left[r(t)\left(D_{-}^{\alpha} y\right)^{\eta}(t)\right]^{\prime}-q(t) f\left(\int_{t}^{\infty}(v-t)^{-\alpha} y(v) d v\right)=0
$$

for $t>0$,

where $D_{-}^{\alpha} y$ denotes the Liouville right-sided fractional derivative of order $\alpha$ with the form

$$
\left(D_{-}^{\alpha} y\right)(t):=-\frac{1}{\Gamma(1-\alpha)} \frac{d}{d t} \int_{t}^{\infty}(v-t)^{-\alpha} y(v) d v
$$

$$
\text { for } t \in \mathbb{R}_{+}:=(0, \infty) \text {, }
$$

and he obtained four main results under the condition of

$$
\begin{gathered}
\int_{t_{0}}^{\infty} r^{-1 / \eta}(t) d t=\infty, \\
\int_{t_{0}}^{\infty} r^{-1 / \eta}(t) d t<\infty
\end{gathered}
$$

by using a generalized Riccati transformation technique and an inequality; see [18].

Using the same method, in 2013, Chen [23] studied oscillatory behavior of the fractional differential equation with the form

$$
\begin{aligned}
& \left(D_{-}^{1+\alpha} y\right)(t)-p(t)\left(D_{-}^{\alpha} y\right)(t) \\
& \quad+q(t) f\left(\int_{t}^{\infty}(v-t)^{-\alpha} y(v) d v\right)=0 \text { for } t>0,
\end{aligned}
$$

where $D_{-}^{\alpha} y$ is the Liouville right-sided fractional derivative of order $\alpha \in(0,1)$ of $y$.

Zheng [24] considered the oscillation of the nonlinear fractional differential equation with damping term:

$$
\begin{aligned}
& {\left[a(t)\left(D_{-}^{\alpha} x(t)\right)^{\gamma}\right]^{\prime}+p(t)\left(D_{-}^{\alpha} x(t)\right)^{\gamma}} \\
& \quad-q(t) f\left(\int_{t}^{\infty}(\xi-t)^{-\alpha} x(\xi) d \xi\right)=0, \quad t \in\left[t_{0}, \infty\right),
\end{aligned}
$$

where $D_{-}^{\alpha} x(t)$ denotes the Liouville right-sided fractional derivative of order $\alpha$ of $x$. Using a generalized Riccati function and inequality technique, he established some new oscillation criteria.

Han et al. [19] considered the oscillation for a class of fractional differential equation:

$$
\begin{array}{r}
{\left[r(t) g\left(\left(D_{-}^{\alpha} y\right)(t)\right)\right]^{\prime}-p(t) f\left(\int_{t}^{\infty}(s-t)^{-\alpha} y(s) d s\right)=0,} \\
\text { for } t>0,
\end{array}
$$

where $0<\alpha<1$ is a real number and $D_{-}^{\alpha} y$ is the Liouville right-sided fractional derivative of order $\alpha$ of $y$.
By generalized Riccati transformation technique, oscillation criteria for the nonlinear fractional differential equation are obtained.

Qi and Cheng [20] studied the oscillation behavior of the equation with the form

$$
\begin{aligned}
(a(t) & {\left.\left[r(t) D_{-}^{\alpha} x(t)\right]^{\prime}\right)^{\prime}+p(t)\left[r(t) D_{-}^{\alpha} x(t)\right]^{\prime} } \\
& -q(t) \int_{t}^{\infty}(\xi-t)^{-\alpha} x(\xi) d \xi=0, \quad t \in\left[t_{0}, \infty\right),
\end{aligned}
$$

where $D_{-}^{\alpha} x(t)$ also denotes the Liouville right-sided fractional derivative and some sufficient conditions for the oscillation of the equation were given.

The above works on the oscillation are all on fractional equations with Liouville right-sided fractional derivative by Riccati transformation technique.

We notice that very little attention is paid to oscillation of fractional differential equations with RiemannLiouville derivative. For the relative works of study for oscillatory behavior of fractional differential equations RiemannLiouville derivative we refer to $[17,21,25,26]$.

Marian et al. [25] presented the oscillatory behavior of forced nonlinear fractional difference equation of the form

$$
\begin{array}{r}
\Delta^{\alpha} x(t)+f_{1}(t, x(t+\alpha))=v(t)+f_{2}(t, x(t+\alpha)), \\
t \in N_{0}, \quad 0<\alpha \leq 1,\left.\quad \Delta^{\alpha-1} x(t)\right|_{t=0}=x_{0},
\end{array}
$$

where $\Delta^{\alpha}$ is a Riemann-Liouville like discrete fractional difference operator of order $\alpha$, and some oscillation criteria are established by the same method with [17].

In 2013, Chen et al. [21] improved and extended some work in [17] by considering the forced oscillation of fractional differential equation:

$$
\begin{gathered}
D_{a}^{q} x+f_{1}(t, x)=v(t)+f_{2}(t, x), \\
\lim _{t \rightarrow a^{+}} J_{a}^{1-q} x(t)=b_{1},
\end{gathered}
$$

with the conditions

$$
\begin{gathered}
D_{a}^{q-k} x(a)=b_{k} \quad(k=1,2, \ldots, m-1), \\
\lim _{t \rightarrow a^{+}} I_{a}^{m-q} x(t)=b_{m},
\end{gathered}
$$

where $D_{a}^{q}$ denotes the Riemann-Liouville or Caputo differential operator of order $q$ with $m-1<q \leq m, m \geq 1$, and the operator $I_{a}^{m-q}$ is the Riemann-Liouville fractional integral operator. The authors obtained some new oscillation criteria by the same method with [17].

In 2014, Wang et al. [26] extended some oscillation results from integer differential equation to the fractional differential equation:

$$
D_{a}^{\alpha} x(t)+q(t) f(x(t))=0, \quad t \in[a,+\infty), a>0,
$$

where $D_{a}^{\alpha}$ denotes the standard Riemann-Liouville differential operator of order $\alpha$ with $0<\alpha \leq 1, q$ is a positive 
real-valued function, $f$ is a continuous functional defined on $[0,+\infty) \rightarrow[0,+\infty)$ satisfying that

$$
\frac{f(x)}{I^{2-\alpha} x} \geq K>0
$$

and $I^{2-\alpha}$ denotes Riemann-Liouville integral operator. The authors obtained some new oscillation criteria by the method of Riccati transformation technique.

The main purpose of this paper is giving several oscillation theorems for the fractional differential equation:

$$
\begin{aligned}
& {\left[a(t)\left(p(t)+q(t)\left(D_{-}^{\alpha} x\right)(t)\right)^{\gamma}\right]^{\prime}} \\
& -b(t) f\left(\int_{t}^{\infty}(s-t)^{-\alpha} x(s) d s\right)=0, \\
& \text { for } t \geqslant t_{0}>0,
\end{aligned}
$$

where $\alpha \in(0,1)$ is a constant, $\gamma>0$ is a quotient of odd positive integers, and $\left(D_{-}^{\alpha} x\right)$ is the Liouville right-sided fractional derivative of order $\alpha$ of $x$ defined by

$$
\left(D_{-}^{\alpha} x\right)(t):=\frac{-1}{\Gamma(1-\alpha)} \frac{d}{d t} \int_{t}^{\infty}(s-t)^{-\alpha} x(s) d s
$$

for $t \in \mathbb{R}_{+}:=(0, \infty)$; here $\Gamma$ is the gamma function defined by $\Gamma(t):=\int_{0}^{\infty} s^{t-1} e^{-s} d s$ for $t \in \mathbb{R}_{+}$, and the following conditions are assumed to hold.

(A) $f: \mathbb{R} \rightarrow \mathbb{R}$ is a continuous function such that $f(\eta) / \eta^{\gamma} \geqslant K$ for a certain constant $K>0$ and for all $\eta \neq 0$. $a, b$, and $q$ are positive continuous functions on $\left[t_{0}, \infty\right)$ for a certain $t_{0}>0$, and $p$ is a nonnegative continuous function on $\left[t_{0}, \infty\right)$ for a certain $t_{0}>0$. There exists $M>0, q(t) \leqslant M$, for $t \in\left[t_{0}, \infty\right)$. And $\int_{t_{0}}^{\infty}(p(t) / q(t)) d t<\infty$.

(B) $(p(t) / q(t))^{\prime} \neq 0$, for $t \in\left[t_{0}, \infty\right)$.

By a solution $x$ of (16) we mean a nontrivial function $x \in C\left(\mathbb{R}_{+}, \mathbb{R}\right)$ such that $\int_{t}^{\infty}(s-t)^{-\alpha} x(s) d s \in C^{1}\left(\mathbb{R}_{+}, \mathbb{R}\right)$ and $a(t)\left(p(t)+q(t)\left(D_{-}^{\alpha} x\right)(t)\right)^{\gamma} \in C^{1}\left(\mathbb{R}_{+}, \mathbb{R}\right)$, satisfying (16) for $t \geqslant$ $t_{0}>0$. We consider only those solutions of (16) that satisfy $\sup \left\{|x(t)|: t>t_{x}\right\}>0$ for any $t_{x} \geqslant t_{0}$. A solution $x$ of (16) is said to be oscillatory if it is neither eventually positive nor eventually negative; otherwise, it is nonoscillatory. Equation (16) is said to be oscillatory if all its solutions are oscillatory.

Our results obtained here improve and extend the main results of [18]. In [18], the author studied the oscillation of (16), where $p(t)=0$ and $q(t)=1$. We are dealing with the oscillation theorems for (16).

For the sake of convenience, we remember

$$
z(t)=p(t)+q(t)\left(D_{-}^{\alpha} x\right)(t) .
$$

\section{Preliminaries and Lemmas}

In this section, we present some useful preliminaries and lemmas, which will be used in the proof of our main results.
Definition 1 (see [28]). The Liouville right-sided fractional integral of order $\sigma>0$ of a function $y: \mathbb{R}_{+} \rightarrow \mathbb{R}$ on the half-axis $\mathbb{R}_{+}$is given by

$$
\left(I_{-}^{\sigma} y\right)(t):=\frac{1}{\Gamma(\sigma)} \int_{t}^{\infty}(s-t)^{\sigma-1} y(s) d s
$$

for $t>0$, provided that the right-hand side is pointwise defined on $\mathbb{R}_{+}$, where $\Gamma$ is the gamma function.

Definition 2 (see [28]). The Liouville right-sided fractional derivative of order $\sigma>0$ of a function $y: \mathbb{R}_{+} \rightarrow \mathbb{R}$ on the half-axis $\mathbb{R}_{+}$is given by

$$
\begin{aligned}
\left(D_{-}^{\sigma} y\right)(t) & :=(-1)^{\lceil\sigma\rceil} \frac{d^{\lceil\sigma\rceil}}{d t^{\lceil\sigma\rceil}}\left(I_{-}^{\lceil\sigma\rceil-\sigma} y\right)(t) \\
& =\frac{(-1)^{\lceil\sigma\rceil}}{\Gamma(\lceil\sigma\rceil-\sigma)} \frac{d^{\lceil\sigma\rceil}}{d t^{\lceil\sigma\rceil}} \int_{t}^{\infty}(s-t)^{\lceil\sigma\rceil-\sigma-1} y(s) d s,
\end{aligned}
$$

for $t>0$,

provided that the right-hand side is pointwise defined on $\mathbb{R}_{+}$, where $\lceil\sigma\rceil:=\min \{z \in \mathbb{Z}: z \geqslant \sigma\}$ is the ceiling function.

Lemma 3 (see [29]). If $A$ and $B$ are nonnegative constants, then

$$
A^{\beta}+(\beta-1) B^{\beta}-\beta A B^{\beta-1} \geqslant 0, \quad \beta>1,
$$

where the equality holds if and only if $A=B$.

Lemma 4 (see [18]). Let $x$ be a solution of (16) and

$$
G(t):=\int_{t}^{\infty}(s-t)^{-\alpha} x(s) d s, \quad \text { for } \alpha \in(0,1), t>0 .
$$

Then

$$
G^{\prime}(t)=-\Gamma(1-\alpha)\left(D_{-}^{\alpha} x\right)(t), \quad \text { for } \alpha \in(0,1), t>0 .
$$

The proof of Lemma 4 is the same as the proof of Lemma 2.1 in [18].

\section{Main Results}

In this section, we establish some new oscillation criteria for (16).

Theorem 5. Assume that (A) holds, and

$$
\int_{t_{0}}^{\infty} a^{-1 / \gamma}(t) d t=\infty .
$$

Furthermore, assume that there exists a positive function $r \in$ $C^{1}\left[t_{0}, \infty\right)$ such that

$$
\begin{aligned}
\limsup _{t \rightarrow \infty} \int_{t_{0}}^{t}[ & \operatorname{Kr}(s) b(s) \\
& \left.\quad-\left(\frac{r_{+}^{\prime}(s)}{r(s)}\right)^{\gamma+1} \frac{M^{\gamma} r(s) a(s)}{(\gamma+1)^{(\gamma+1)} \Gamma^{\gamma}(1-\alpha)}\right] d s=\infty,
\end{aligned}
$$


where $r_{+}^{\prime}(s):=\max \left\{r^{\prime}(s), 0\right\}$. Then every solution of (16) is oscillatory.

Proof. Suppose that $x(t)$ is a nonoscillatory solution of (16). Without loss of generality, we may assume that $x(t)$ is an eventually positive solution of (16). Then there exists $t_{1} \in$ $\left[t_{0}, \infty\right)$ such that

$$
x(t)>0, \quad G(t)>0, \quad \text { for } t \in\left[t_{1}, \infty\right),
$$

where $G(t)$ is defined as in (22). Therefore, it follows from (16) that

$$
\left[a(t) z^{\gamma}(t)\right]^{\prime}=b(t) f(G(t))>0, \quad \text { for } t \in\left[t_{1}, \infty\right) .
$$

Thus, $a(t) z^{\gamma}(t)$ is strictly increasing on $\left[t_{1}, \infty\right)$ and is eventually of one sign. Since $a(t)>0$ for $t \in\left[t_{0}, \infty\right)$ and $\gamma>0$ is a quotient of odd positive integers, we see that $z(t)$ is eventually of one sign. We first show

$$
z(t)<0, \quad \text { for } t \in\left[t_{1}, \infty\right) .
$$

Otherwise, there exists $t_{2} \geqslant t_{1}$ such that $z\left(t_{2}\right)>0$, and since $a(t) z^{\gamma}(t)$ is strictly increasing on $\left[t_{1}, \infty\right)$, it is clear that $a(t) z^{\gamma}(t) \geqslant a\left(t_{2}\right) z^{\gamma}\left(t_{2}\right):=c_{1}>0$ for $t \in\left[t_{2}, \infty\right)$. Therefore, we have

$$
z(t) \geqslant c_{1}^{1 / \gamma} a^{-1 / \gamma}(t) .
$$

Due to $q(t)>0$, from (18), we get

$$
\frac{p(t)}{q(t)}+\left(D_{-}^{\alpha} x\right)(t) \geqslant \frac{c_{1}^{1 / \gamma} a^{-1 / \gamma}(t)}{q(t)} \geqslant \frac{c_{1}^{1 / \gamma} a^{-1 / \gamma}(t)}{M} .
$$

Integrating both sides of last inequality from $t_{2}$ to $t$, from (23), we obtain

$$
\int_{t_{2}}^{t}\left(\frac{p(s)}{q(s)}-\frac{G^{\prime}(s)}{\Gamma(1-\alpha)}\right) d s \geqslant \int_{t_{2}}^{t} \frac{c_{1}^{1 / \gamma} a^{-1 / \gamma}(s)}{M} d s .
$$

So, we get

$$
\begin{aligned}
G(t) \leqslant & G\left(t_{2}\right)+\Gamma(1-\alpha) \\
& \times\left(\int_{t_{2}}^{t} \frac{p(s)}{q(s)} d s-\frac{c_{1}^{1 / \gamma}}{M} \int_{t_{2}}^{t} a^{-1 / \gamma}(s) d s\right) \\
\text { as } t & \longrightarrow \infty,-\infty,
\end{aligned}
$$

and this contradicts (26). Hence, we have that (28) holds.

From (A), (18), and (23), we have

$$
z(t)=p(t)+q(t)\left(D_{-}^{\alpha} x\right)(t)=p(t)+q(t)\left(-\frac{G^{\prime}(t)}{\Gamma(1-\alpha)}\right) .
$$

Therefore,

$$
\begin{aligned}
G^{\prime}(t) & =\Gamma(1-\alpha) \frac{p(t)-z(t)}{q(t)} \\
& \geqslant-\Gamma(1-\alpha) \frac{z(t)}{q(t)} \geqslant-\Gamma(1-\alpha) \frac{z(t)}{M} .
\end{aligned}
$$

Define the function $w(t)$ by a generalized Riccati transformation

$$
w(t)=r(t) \frac{-a(t) z^{\gamma}(t)}{G^{\gamma}(t)}, \quad \text { for } t \in\left[t_{1}, \infty\right)
$$

Then, we have $w(t)>0$ for $t \in\left[t_{0}, \infty\right)$, and from (16), (34), (35), and (A), it follows that

$$
\begin{aligned}
w^{\prime}(t)= & r^{\prime}(t) \frac{-a(t) z^{\gamma}(t)}{G^{\gamma}(t)}+r(t)\left[\frac{-a(t) z^{\gamma}(t)}{G^{\gamma}(t)}\right]^{\prime} \\
= & \frac{r^{\prime}(t)}{r(t)} w(t)-r(t) \frac{b(t) f(G(t))}{G^{\gamma}(t)} \\
& +\gamma r(t) a(t) z^{\gamma}(t) \frac{G^{\prime}(t)}{G^{\gamma+1}(t)} \\
\leqslant & \frac{r_{+}^{\prime}(t)}{r(t)} w(t)-\frac{\gamma r(t) a(t) \Gamma(1-\alpha)}{M} \frac{z^{\gamma+1}(t)}{G^{\gamma+1}(t)} \\
& -\operatorname{Kr}(t) b(t) \\
= & \frac{r_{+}^{\prime}(t)}{r(t)} w(t)-\frac{\gamma \Gamma(1-\alpha)}{M[r(t) a(t)]^{1 / \gamma}} w^{1+1 / \gamma}(t) \\
& -\operatorname{Kr}(t) b(t), \quad \text { for } t \in\left[t_{1}, \infty\right),
\end{aligned}
$$

where $r_{+}^{\prime}(t)$ is defined as in Theorem 5. Let

$$
\begin{gathered}
\beta=1+\frac{1}{\gamma}, \quad A=\left(\frac{\gamma \Gamma(1-\alpha)}{M(r(t) a(t))^{1 / \gamma}}\right)^{1 / \beta} w(t), \\
B=\left(\frac{r_{+}^{\prime}(t)}{r(t)}\right)^{\gamma} \frac{M^{\gamma / \beta}[r(t) a(t)]^{1 / \beta}}{\beta^{\gamma}[\gamma \Gamma(1-\alpha)]^{\gamma / \beta}} .
\end{gathered}
$$

From (21) and (36), we derive

$$
\begin{array}{r}
w^{\prime}(t) \leqslant-\operatorname{Kr}(t) b(t)+\left(\frac{r_{+}^{\prime}(t)}{r(t)}\right)^{\gamma+1} \frac{M^{\gamma} r(t) a(t)}{(\gamma+1)^{(\gamma+1)} \Gamma^{\gamma}(1-\alpha)}, \\
\text { for } t \in\left[t_{1}, \infty\right) .
\end{array}
$$

Integrating both sides of (38) from $t_{1}$ to $t$, we have

$$
\begin{gathered}
\int_{t_{1}}^{t}\left[\operatorname{Kr}(s) b(s)-\left(\frac{r_{+}^{\prime}(s)}{r(s)}\right)^{\gamma+1} \frac{M^{\gamma} r(s) a(s)}{(\gamma+1)^{(\gamma+1)} \Gamma^{\gamma}(1-\alpha)}\right] d s \\
\leqslant w\left(t_{1}\right)-w(t)<w\left(t_{1}\right), \quad \text { for } t \in\left[t_{1}, \infty\right) .
\end{gathered}
$$


Letting $t \rightarrow \infty$, we get

$$
\begin{aligned}
\underset{t \rightarrow \infty}{\limsup } \int_{t_{1}}^{t} & {[\operatorname{Kr}(s) b(s)} \\
& \left.\quad-\left(\frac{r_{+}^{\prime}(s)}{r(s)}\right)^{\gamma+1} \frac{M^{\gamma} r(s) a(s)}{(\gamma+1)^{(\gamma+1)} \Gamma^{\gamma}(1-\alpha)}\right] d s \\
\leqslant w\left(t_{1}\right)<\infty &
\end{aligned}
$$

which contradicts (25). The proof is complete.

Theorem 6. Suppose that (A) and (24) hold. Furthermore, suppose that there exists a positive function $r \in C^{1}\left[t_{0}, \infty\right)$, and a function $H \in C(\mathbb{Q}, \mathbb{R})$, where $\mathbb{\square}:=\left\{(s, t): s \geqslant t \geqslant t_{0}\right\}$, such that

$$
\begin{gathered}
H(t, t)=0, \quad \text { for } t \geqslant t_{0}, \\
H(s, t)>0, \quad \text { for }(s, t) \in \mathbb{\mathbb { V }}_{0},
\end{gathered}
$$

where $\square_{0}:=\left\{(s, t): s>t \geqslant t_{0}\right\}$, and $H$ has a nonpositive continuous partial derivate $H_{t}^{\prime}(s, t)=\partial H(s, t) / \partial t$ on $\rrbracket_{0}$, with respect to the second variable, and satisfies

$$
\begin{aligned}
\limsup _{s \rightarrow \infty} & \frac{1}{H\left(s, t_{0}\right)} \\
\times \int_{t_{0}}^{s-1} & {[\operatorname{Kr}(t) b(t) H(s, t)} \\
& \left.\quad-\frac{M^{\gamma} r(t) a(t) h_{+}^{\gamma+1}(s, t)}{(\gamma+1)^{\gamma+1}[\Gamma(1-\alpha) H(s, t)]^{\gamma}}\right] d t=\infty
\end{aligned}
$$

where $h_{+}(s, t)=\max \left\{0, H_{t}^{\prime}(s, t)+\left(r_{+}^{\prime}(t) / r(t)\right) H(s, t)\right\}$ for $(s, t) \in \mathbb{\square}_{0}$; here $r_{+}^{\prime}(t)$ is defined as in Theorem 5. Then all solutions of (16) are oscillatory.

Proof. Suppose that $x(t)$ is a nonoscillatory solution of (16). Without loss of generality, we may assume that $x(t)$ is an eventually positive solution of (16). We proceed as in proof of Theorem 5 to get that (36) holds. Multiplying (36) by $H(s, t)$ and integrating from $t_{1}$ to $s-1$, for $s \in\left[t_{1}+1, \infty\right)$, we derive

$$
\begin{aligned}
& \int_{t_{1}}^{s-1} \operatorname{Kr}(t) b(t) H(s, t) d t \\
& \leqslant-\int_{t_{1}}^{s-1} H(s, t) w^{\prime}(t) d t+\int_{t_{1}}^{s-1} \frac{r_{+}^{\prime}(t)}{r(t)} H(s, t) w(t) d t \\
& -\int_{t_{1}}^{s-1} H(s, t) \frac{\gamma \Gamma(1-\alpha) w^{1+1 / \gamma}(t)}{M[r(t) a(t)]^{1 / \gamma}} d t, \\
& \text { for } s \in\left[t_{1}+1, \infty\right) .
\end{aligned}
$$

From

$$
\begin{aligned}
& -\int_{t_{1}}^{s-1} H(s, t) w^{\prime}(t) d t \\
& =[-H(s, t) w(t)]_{t=t_{1}}^{t=s-1}+\int_{t_{1}}^{s-1} H_{t}^{\prime}(s, t) w(t) d t \\
& \leqslant H\left(s, t_{1}\right) w\left(t_{1}\right)+\int_{t_{1}}^{s-1} H_{t}^{\prime}(s, t) w(t) d t \\
& \quad \text { for } s \in\left[t_{1}+1, \infty\right),
\end{aligned}
$$

we have

$$
\begin{aligned}
& \int_{t_{1}}^{s-1} \operatorname{Kr}(t) b(t) H(s, t) d t \\
& \leqslant H\left(s, t_{1}\right) w\left(t_{1}\right) \\
& +\int_{t_{1}}^{s-1}\left\{\left[H_{t}^{\prime}(s, t)+\frac{r_{+}^{\prime}(t)}{r(t)} H(s, t)\right] w(t)\right. \\
& \left.\quad-\frac{\gamma \Gamma(1-\alpha) H(s, t)}{M[r(t) a(t)]^{1 / \gamma}} w^{1+1 / \gamma}(t)\right\} d t \\
& +\int_{t_{1}}^{s-1}\left[\begin{array}{c}
\left.h_{+}, t, t\right) w(t) \\
\left.-\frac{\gamma \Gamma(1-\alpha) H(s, t)}{M[r(t) a(t)]^{1 / \gamma}} w^{(1+\gamma) / \gamma}(t)\right] d t \\
\text { for } s \in\left[t_{1}+1, \infty\right),
\end{array}\right.
\end{aligned}
$$

where $h_{+}(t)$ is defined as in Theorem 6. Let

$$
\begin{gathered}
\beta=1+\frac{1}{\gamma}, \quad A=\left[\frac{\gamma \Gamma(1-\alpha) H(s, t)}{M[r(t) a(t)]^{1 / \gamma}}\right]^{1 / \beta} w(t), \\
B=\left[\frac{h_{+}(s, t)}{\beta}\right]^{\gamma} \frac{M^{\gamma / \beta}[r(t) a(t)]^{1 / \beta}}{[\gamma \Gamma(1-\alpha) H(s, t)]^{\gamma / \beta}} .
\end{gathered}
$$

From (21) and (45), we get

$$
\begin{aligned}
\int_{t_{1}}^{s-1} & \operatorname{Kr}(t) b(t) H(s, t) d t \\
\leqslant & H\left(s, t_{1}\right) w\left(t_{1}\right) \\
& \quad+\int_{t_{1}}^{s-1} \frac{M^{\gamma} r(t) a(t) h_{+}^{\gamma+1}(s, t)}{(\gamma+1)^{\gamma+1}[\Gamma(1-\alpha) H(s, t)]^{\gamma}} d t .
\end{aligned}
$$

From $H_{t}^{\prime}(s, t)<0$, for $s>t \geqslant t_{0}$, we have

$$
0<H\left(s, t_{1}\right) \leqslant H\left(s, t_{0}\right), \quad \text { for } s>t_{1} \geqslant t_{0},
$$

and $0<H(s, t) \leqslant H\left(s, t_{0}\right)$, for $s>t \geqslant t_{0}$; then

$$
0<\frac{H(s, t)}{H\left(s, t_{0}\right)} \leqslant 1, \quad \text { for } s>t \geqslant t_{0} .
$$


Therefore, we get

$$
\begin{aligned}
& \frac{1}{H\left(s, t_{0}\right)} \int_{t_{0}}^{s-1}[\operatorname{Kr}(t) b(t) H(s, t) \\
& \left.-\frac{M^{\gamma} r(t) a(t) h_{+}^{\gamma+1}(s, t)}{(\gamma+1)^{\gamma+1}[\Gamma(1-\alpha) H(s, t)]^{\gamma}}\right] d t \\
& =\frac{1}{H\left(s, t_{0}\right)} \int_{t_{0}}^{t_{1}}[\operatorname{Kr}(t) b(t) H(s, t) \\
& \left.-\frac{M^{\gamma} r(t) a(t) h_{+}^{\gamma+1}(s, t)}{(\gamma+1)^{\gamma+1}[\Gamma(1-\alpha) H(s, t)]^{\gamma}}\right] d t \\
& +\frac{1}{H\left(s, t_{0}\right)} \int_{t_{1}}^{s-1}[\operatorname{Kr}(t) b(t) H(s, t) \\
& \left.-\frac{M^{\gamma} r(t) a(t) h_{+}^{\gamma+1}(s, t)}{(\gamma+1)^{\gamma+1}[\Gamma(1-\alpha) H(s, t)]^{\gamma}}\right] d t \\
& \leqslant \frac{1}{H\left(s, t_{0}\right)} \int_{t_{0}}^{t_{1}} \operatorname{Kr}(t) b(t) H(s, t) d t \\
& +\frac{1}{H\left(s, t_{0}\right)} H\left(s, t_{1}\right) w\left(t_{1}\right) \\
& \leqslant \int_{t_{0}}^{t_{1}} \operatorname{Kr}(t) b(t) d t+w\left(t_{1}\right), \quad \text { for } s \in\left[t_{1}+1, \infty\right) .
\end{aligned}
$$

Letting $s \rightarrow \infty$, we get

$$
\begin{aligned}
& \limsup _{s \rightarrow \infty} \frac{1}{H\left(s, t_{0}\right)} \\
& \quad \times \int_{t_{0}}^{s-1}[\operatorname{Kr}(t) b(t) H(s, t) \\
& \left.\quad-\frac{M^{\gamma} r(t) a(t) h_{+}^{\gamma+1}(s, t)}{(\gamma+1)^{\gamma+1}[\Gamma(1-\alpha) H(s, t)]^{\gamma}}\right] d t \\
& \leqslant \int_{t_{0}}^{t_{1}} \operatorname{Kr}(t) b(t) d t+w\left(t_{1}\right)<\infty,
\end{aligned}
$$

which is a contradiction to (42). The proof is complete.

Next, we consider the condition of

$$
\int_{t_{0}}^{\infty} a^{-1 / \gamma}(t) d t<\infty,
$$

which yields that (24) does not hold. Under this condition, we have the following results.
Theorem 7. Suppose that (A), (B), and (52) hold, and there exists a positive function $r \in C^{1}\left[t_{0}, \infty\right)$ such that (25) holds. Furthermore, assume that, for every constant $T \geqslant t_{0}$,

$$
\int_{T}^{\infty}\left[\frac{1}{a(t)} \int_{T}^{t} b(s) d s\right]^{1 / \gamma} d t=\infty .
$$

Then every solution $x$ of (16) is oscillatory or satisfies $\lim _{t \rightarrow \infty} G^{\prime}(t)=0$ or $\lim _{t \rightarrow \infty} G(t)=0$, where $G(t)$ is defined as Lemma 4.

Proof. Assume that $x(t)$ is a nonoscillatory solution of (16). Without loss of generality, assume that $x(t)$ is an eventually positive solution of (16). Proceeding as in the proof of Theorem 5, we get that (26) and (27) hold. Then there are two cases for the sign of $z(t)$.

When $z(t)$ is eventually negative, from the proof of Theorem 5, we get that every solution $x(t)$ of (16) is oscillatory.

Next, assume that $z(t)$ is eventually positive; then there exists $t_{21} \geqslant t_{0}$, such that $z(t)>0$, for $t \geqslant t_{21}$. From (18) and (23), we get

$$
\begin{gathered}
z(t)=p(t)+q(t)\left(D_{-}^{\alpha} x\right)(t)=p(t) \\
+q(t)\left(-\frac{G^{\prime}(t)}{\Gamma(1-\alpha)}\right)>0 .
\end{gathered}
$$

Therefore,

$$
G^{\prime}(t)<\Gamma(1-\alpha) \frac{p(t)}{q(t)} .
$$

Since (B) holds and $\int_{t_{0}}^{\infty}(p(t) / q(t)) d t<\infty$, then we get

$$
\lim _{t \rightarrow \infty} \frac{p(t)}{q(t)}=0 \text {. }
$$

Letting $t \rightarrow \infty$ in (55), we have

$$
\lim _{t \rightarrow \infty} G^{\prime}(t) \leqslant 0
$$

If $\lim _{t \rightarrow \infty} G^{\prime}(t)<0$, then there exists $t_{22} \geqslant t_{1}$ such that $G^{\prime}(t)<0$, for $t \geqslant t_{22}$. We set $t_{2}:=\max \left\{t_{21}, t_{22}\right\}$. Thus, we get $\lim _{t \rightarrow \infty} G(t)=C$, and $G(t) \geqslant C, t \geq t_{2}$.

We now prove $C=0$. If not, that is, $C>0$, then from (27), we derive

$$
\begin{array}{r}
{\left[a(t) z^{\gamma}(t)\right]^{\prime}=b(t) f(G(t)) \geqslant K b(t) G^{\gamma}(t) \geqslant K C^{\gamma} b(t),} \\
\text { for } t \in\left[t_{2}, \infty\right) .
\end{array}
$$

Integrating both sides of (58) from $t_{2}$ to $t$, we have

$$
\begin{aligned}
a(t) z^{\gamma}(t) & \geqslant a\left(t_{2}\right) z^{\gamma}\left(t_{2}\right)+K C^{\gamma} \int_{t_{2}}^{t} b(s) d s \\
& >K C^{\gamma} \int_{t_{2}}^{t} b(s) d s, \quad \text { for } t \in\left[t_{2}, \infty\right) .
\end{aligned}
$$


Therefore,

$$
z(t)>C K^{1 / \gamma}\left[\frac{1}{a(t)} \int_{t_{2}}^{t} b(s) d s\right]^{1 / \gamma}
$$

Hence, from (18), (A), and (23), we get

$$
\begin{aligned}
\frac{p(t)}{q(t)}-\frac{G^{\prime}(t)}{\Gamma(1-\alpha)} & >\frac{C K^{1 / \gamma}}{q(t)}\left[\frac{1}{a(t)} \int_{t_{2}}^{t} b(s) d s\right]^{1 / \gamma} \\
& >\frac{C K^{1 / \gamma}}{M}\left[\frac{1}{a(t)} \int_{t_{2}}^{t} b(s) d s\right]^{1 / \gamma} .
\end{aligned}
$$

Integrating both sides of (61) from $t_{2}$ to $t$, we have

$$
\begin{aligned}
\int_{t_{2}}^{t} \frac{p(s)}{q(s)} d s-\frac{G(t)-G\left(t_{2}\right)}{\Gamma(1-\alpha)} & \\
& >\frac{C K^{1 / \gamma}}{M} \int_{t_{2}}^{t}\left[\frac{1}{a(v)} \int_{t_{2}}^{v} b(s) d s\right]^{1 / \gamma} d v .
\end{aligned}
$$

Then, we obtain

$$
\begin{aligned}
G(t)< & \left\{\int_{t_{2}}^{t} \frac{p(s)}{q(s)} d s-\frac{C K^{1 / \gamma}}{M}\right. \\
& \left.\times \int_{t_{2}}^{t}\left[\frac{1}{a(v)} \int_{t_{2}}^{v} b(s) d s\right]^{1 / \gamma} d v\right\} \Gamma(1-\alpha)+G\left(t_{2}\right) \\
& \longrightarrow-\infty, \quad \text { as } t \longrightarrow \infty .
\end{aligned}
$$

This contradicts (26). Therefore, we have $C=0$; that is,

$$
\lim _{t \rightarrow \infty} G(t)=0
$$

The proof is complete.

Theorem 8. Suppose that (A), (B), and (52) hold. Let $r(t)$ and $H(s, t)$ be defined as in Theorem 6 such that (42) holds. Furthermore, assume that, for every $T \geqslant t_{0},(53)$ holds. Then every solution $x$ of $(16)$ is oscillatory or satisfies $\lim _{t \rightarrow \infty} G^{\prime}(t)=0$ or $\lim _{t \rightarrow \infty} G(t)=0$, where $G(t)$ is defined as in Lemma 4 .

Proof. Assume that $x$ is a nonoscillatory solution of (16). Without loss of generality, assume that $x$ is an eventually positive solution of (16). Proceeding as in the proof of Theorem 5, we get that (26) and (27) hold. Then there are two cases for the sign of $z(t)$.

When $z(t)$ is eventually negative, the proof is similar to that of Theorem 6 . When $z(t)$ is eventually positive, the proof is similar to that of Theorem 7 . Here we omitted it.

Remark 9. From Theorems 5-8, we can get many different sufficient conditions for the oscillation of (16) with different choices of the functions $r$ and $H$.

\section{Examples}

Example 10. Consider the differential equation with fractional-order derivatives:

$$
\begin{aligned}
& {\left[t^{\gamma-2}\left(e^{-t}+\frac{1}{t}\left(D_{-}^{\alpha} x\right)(t)\right)^{\gamma}\right]^{\prime}} \\
& \quad-\frac{1}{t^{2}}\left(\int_{t}^{\infty}(s-t)^{-\alpha} x(s) d s\right)^{\gamma}=0, \quad t \geqslant 1,
\end{aligned}
$$

where $\alpha \in(0,1)$, and $\gamma>0$ is a quotient of odd positive integers.

Here, $a(t)=t^{\gamma-2}, b(t)=1 / t^{2}, p(t)=e^{-t}$, and $q(t)=1 / t$. Take $t_{0}=1, K=1$, and $M=1$. From

$$
\begin{gathered}
\int_{t_{0}}^{\infty} a^{-1 / \gamma}(t) d t=\int_{t_{0}}^{\infty} \frac{1}{t^{1-(2 / \gamma)}} d t=\infty, \\
\int_{t_{0}}^{\infty} \frac{p(t)}{q(t)} d t=\int_{t_{0}}^{\infty} \frac{e^{-t}}{1 / t} d t=\int_{t_{0}}^{\infty} t e^{-t} d t<\infty,
\end{gathered}
$$

we see that (A) and (24) hold. Letting $r(s)=s$, we get

$$
\begin{aligned}
& \limsup _{t \rightarrow \infty} \int_{t_{0}}^{t}[\operatorname{Kr}(s) b(s) \\
& \left.-\left(\frac{r_{+}^{\prime}(s)}{r(s)}\right)^{\gamma+1} \frac{M^{\gamma} r(s) a(s)}{(\gamma+1)^{(\gamma+1)} \Gamma^{\gamma}(1-\alpha)}\right] d s \\
& =\limsup _{t \rightarrow \infty} \int_{t_{0}}^{t}\left[s \cdot \frac{1}{s^{2}}-\left(\frac{1}{s}\right)^{\gamma+1} \frac{s \cdot s^{\gamma-2}}{(\gamma+1)^{\gamma+1} \Gamma^{\gamma}(1-\alpha)}\right] d s \\
& =\limsup _{t \rightarrow \infty} \int_{t_{0}}^{t}\left[\frac{1}{s}-\frac{1}{(\gamma+1)^{\gamma+1} \Gamma^{\gamma}(1-\alpha)} \cdot \frac{1}{s^{2}}\right] d s=\infty \text {, }
\end{aligned}
$$

which satisfies condition (25). Therefore, by Theorem 5 , every solution of (65) is oscillatory.

Example 11. Consider the differential equation with fractional-order derivatives:

$$
\begin{aligned}
& {\left[t^{\gamma+2}\left(\frac{1}{t^{3}}+\frac{1}{t}\left(D_{-}^{\alpha} x\right)(t)\right)^{\gamma}\right]^{\prime}} \\
& \quad-6 t\left(2+\left(\int_{t}^{\infty}(s-t)^{-\alpha} x(s) d s\right)^{2}\right) \\
& \quad \times\left(\int_{t}^{\infty}(s-t)^{-\alpha} x(s) d s\right)^{\gamma}=0, \quad t \geqslant 2,
\end{aligned}
$$

where $\alpha \in(0,1)$, and $\gamma>0$ is a quotient of odd positive integers. 
Here, $a(t)=t^{2+\gamma}, b(t)=6 t, p(t)=1 / t^{3}, q(t)=1 / t$, and $f(t)=\left(2+t^{2}\right) t^{\gamma}$. Take $K=2, M=1$ and $t_{0}=2, f(u)=u$. From

$$
\begin{gathered}
\int_{t_{0}}^{\infty} a^{-1 / \gamma}(t) d t=\int_{t_{0}}^{\infty} \frac{1}{t^{1+(2 / \gamma)}} d t<\infty, \\
\left(\frac{p(t)}{q(t)}\right)^{\prime}=\left(\frac{1 / t^{3}}{1 / t}\right)^{\prime}=-\frac{2}{t^{3}} \neq 0, \\
\int_{t_{0}}^{\infty} \frac{p(t)}{q(t)} d t=\int_{t_{0}}^{\infty} \frac{1}{t^{2}} d t<\infty
\end{gathered}
$$

we find that (A), (B), and (52) hold.

Take $r(s) \equiv 1$, we have

$$
\begin{aligned}
& \limsup _{t \rightarrow \infty} \int_{t_{0}}^{t}[\operatorname{Kr}(s) b(s) \\
& \left.\quad-\left(\frac{r_{+}^{\prime}(s)}{r(s)}\right)^{\gamma+1} \frac{M^{\gamma} r(s) a(s)}{(\gamma+1)^{(\gamma+1)} \Gamma^{\gamma}(1-\alpha)}\right] d s \\
& \quad=\limsup _{t \rightarrow \infty} \int_{t_{0}}^{t} 12 s d s=\infty,
\end{aligned}
$$

which satisfies condition (25). For every constant $T \geqslant t_{0}, t \in$ $[2 T, \infty)$, we obtain

$$
\begin{aligned}
\int_{T}^{\infty} & {\left[\frac{1}{a(t)} \int_{T}^{t} b(s) d s\right]^{1 / \gamma} d t } \\
& =\int_{T}^{\infty}\left[\frac{1}{t^{\gamma+2}} \int_{T}^{t} 6 s d s\right]^{1 / \gamma} d t \\
& =\int_{T}^{\infty}\left[\frac{3 t^{2}-3 T^{2}}{t^{2+\gamma}}\right]^{1 / \gamma} d t \\
& \geqslant \int_{T}^{\infty} \frac{2^{1 / \gamma}}{t} d t=\infty,
\end{aligned}
$$

which implies that (53) holds. Therefore, by Theorem 7, every solution $x$ of (68) is oscillatory or satisfies $\lim _{t \rightarrow \infty} G^{\prime}(t)=0$ or $\lim _{t \rightarrow \infty} G(t)=0$.

\section{Conflict of Interests}

The authors declare that there is no conflict of interests regarding the publication of this paper.

\section{Acknowledgments}

The authors sincerely thank the reviewers for their valuable suggestions and useful comments that have led to the present improved version of the original paper. This research is supported by the Natural Science Foundation of China (61374074), Natural Science Outstanding Youth Foundation of Shandong Province (JQ201119), and Shandong Provincial Natural Science Foundation (ZR2012AM009 and ZR2013AL003).

\section{References}

[1] Y. A. Rossikhin and M. V. Shitikova, "Applications of fractional calculus to dynamic problems of linear and nonlinear hereditary mechanics of solids," Applied Mechanics Reviews, vol. 50, no. 1, pp. 15-67, 1997.

[2] W. G. Glöckle and T. F. Nonnenmacher, "A fractional calculus approach to self-similar protein dynamics," Biophysical Journal, vol. 68, no. 1, pp. 46-53, 1995.

[3] R. L. Magin, "Fractional calculus in bioengineering," Critical Reviews in Biomedical Engineering, vol. 32, no. 1, pp. 1-377, 2004.

[4] R. L. Bagley and P. J. Torvik, "A theoretical basis for the application of fractional calilus to visoelasticity," Journal of Rheology, vol. 27, no. 3, pp. 201-210, 1983.

[5] R. T. Baillie, "Long memory processes and fractional integration in econometrics," Journal of Econometrics, vol. 73, no. 1, pp. 559, 1996.

[6] F. Mainardi, "Fractional calculus: some basic problems in continuum and statistical mechanics," in Fractals and Fractionals Caluculus in Continuum Mechanics, A. Capinteri and F. Mainardi, Eds., vol. 378, pp. 291-348, Springer, New York, NY, USA, 1997.

[7] B. Mandelbrot, "Some noises with $1 / f$ spectrum, a bridge between direct current and white noise," IEEE Transactions on Information Theory, vol. 13, pp. 289-298, 1967.

[8] I. Podlubny, Fractional Differential Equations, Academic Press, San Diego, Calif, USA, 1999.

[9] Y. Zhou, F. Jiao, and J. Li, "Existence and uniqueness for $p$-type fractional neutral differential equations," Nonlinear Analysis: Theory, Methods and Applications, vol. 71, no. 7-8, pp. 27242733, 2009.

[10] W. Deng, "Smoothness and stability of the solutions for nonlinear fractional differential equations," Nonlinear Analysis: Theory, Methods \& Applications A, vol. 72, no. 3-4, pp. 1768-1777, 2010.

[11] L. Galeone and R. Garrappa, "Explicit methods for fractional differential equations and their stability properties," Journal of Computational and Applied Mathematics, vol. 228, no. 2, pp. 548-560, 2009.

[12] M. Muslim, "Existence and approximation of solutions to fractional differential equations," Mathematical and Computer Modelling, vol. 49, no. 5-6, pp. 1164-1172, 2009.

[13] F. Ghoreishi and S. Yazdani, "An extension of the spectral Tau method for numerical solution of multi-order fractional differential equations with convergence analysis," Computers \& Mathematics with Applications, vol. 61, no. 1, pp. 30-43, 2011.

[14] J. T. Edwards, N. J. Ford, and A. C. Simpson, "The numerical solution of linear multi-term fractional differential equations: systems of equations," Journal of Computational and Applied Mathematics, vol. 148, no. 2, pp. 401-418, 2002.

[15] A. Saadatmandi and M. Dehghan, "A new operational matrix for solving fractional-order differential equations," Computers and Mathematics with Applications, vol. 59, no. 3, pp. 1326-1336, 2010.

[16] J. C. Trigeassou, N. Maamri, J. Sabatier, and A. Oustaloup, "A Lyapunov approach to the stability of fractional differential equations," Signal Processing, vol. 91, no. 3, pp. 437-445, 2011.

[17] S. R. Grace, R. P. Agarwal, P. J. Y. Wong, and A. Zafer, "On the oscillation of fractional differential equations," Fractional Calculus and Applied Analysis, vol. 15, no. 2, pp. 222-231, 2012. 
[18] D.-X. Chen, "Oscillation criteria of fractional differential equations," Advances in Difference Equations, vol. 2012, article 33, 18 pages, 2012.

[19] Z. Han, Y. Zhao, Y. Sun, and C. Zhang, "Oscillation for a class of fractional differential equation," Discrete Dynamics in Nature and Society, vol. 2013, Article ID 390282, 6 pages, 2013.

[20] C. Qi and J. Cheng, "Interval oscillation criteria for a class of fractional differential equations with damping term," Mathematical Problems in Engineering, vol. 2013, Article ID 301085, 8 pages, 2013.

[21] D. Chen, P. Qu, and Y. Lan, "Forced oscillation of certain fractional differential equations," Advances in Difference Equations, vol. 2013, article 125, pp. 1-10, 2013.

[22] S. L. Marian, "Oscillation of fractional nonlinear difference equations," Mathematica Aeterna, vol. 2, no. 9-10, pp. 805-813, 2012.

[23] D. Chen, "Oscillatory behavior of a class of fractional differential equations with damping," UPB Scientific Bulletin, vol. 75, no. 1, pp. 107-118, 2013.

[24] B. Zheng, "Oscillation for a class of nonlinear fractional differential equations with damping term," Journal of Advanced Mathematical Studies, vol. 6, no. 1, pp. 107-115, 2013.

[25] S. L. Marian, M. R. Sagayaraj, A. G. M. Selvam, and M. P. Loganathan, "Oscillation of fractional nonlinear difference equations," Mathematica Aeterna, vol. 2, no. 9-10, pp. 805-813, 2012.

[26] Y. Wang, Z. Han, P. Zhao, and S. Sun, "On the oscillation and asymptotic behavior for a kind of fractional differential equations," Advances in Difference Equations, vol. 2014, article $50,2014$.

[27] Y.-Z. Wang, Z.-L. Han, and S. R. Sun, "Oscillation theorem for a kind of fractional differential equations," Journal of Binzhou University, vol. 29, no. 6, pp. 18-22, 2013.

[28] A. A. Kilbas, H. M. Srivastava, and J. J. Trujillo, Theory and Applications of Fractional Differential Equations, vol. 204 of Elsevier Science BV, Amsterdam, The Netherlands, 2006.

[29] G. H. Hardy, J. E. Littlewood, and G. Polya, Inequalities, Cambridge University Press, Cambridge, UK, 2nd edition, 1998. 


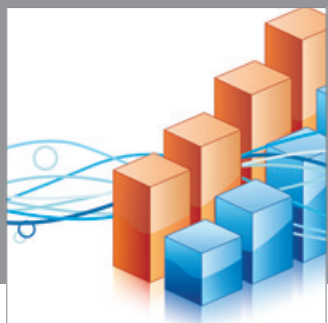

Advances in

Operations Research

mansans

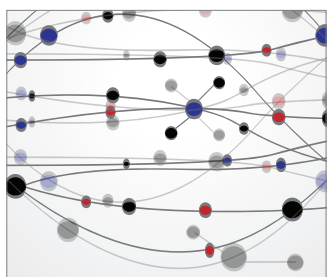

The Scientific World Journal
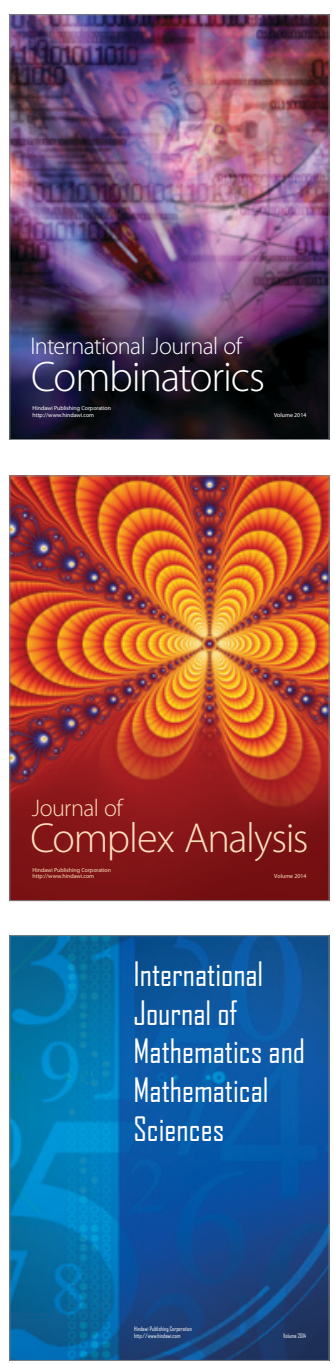
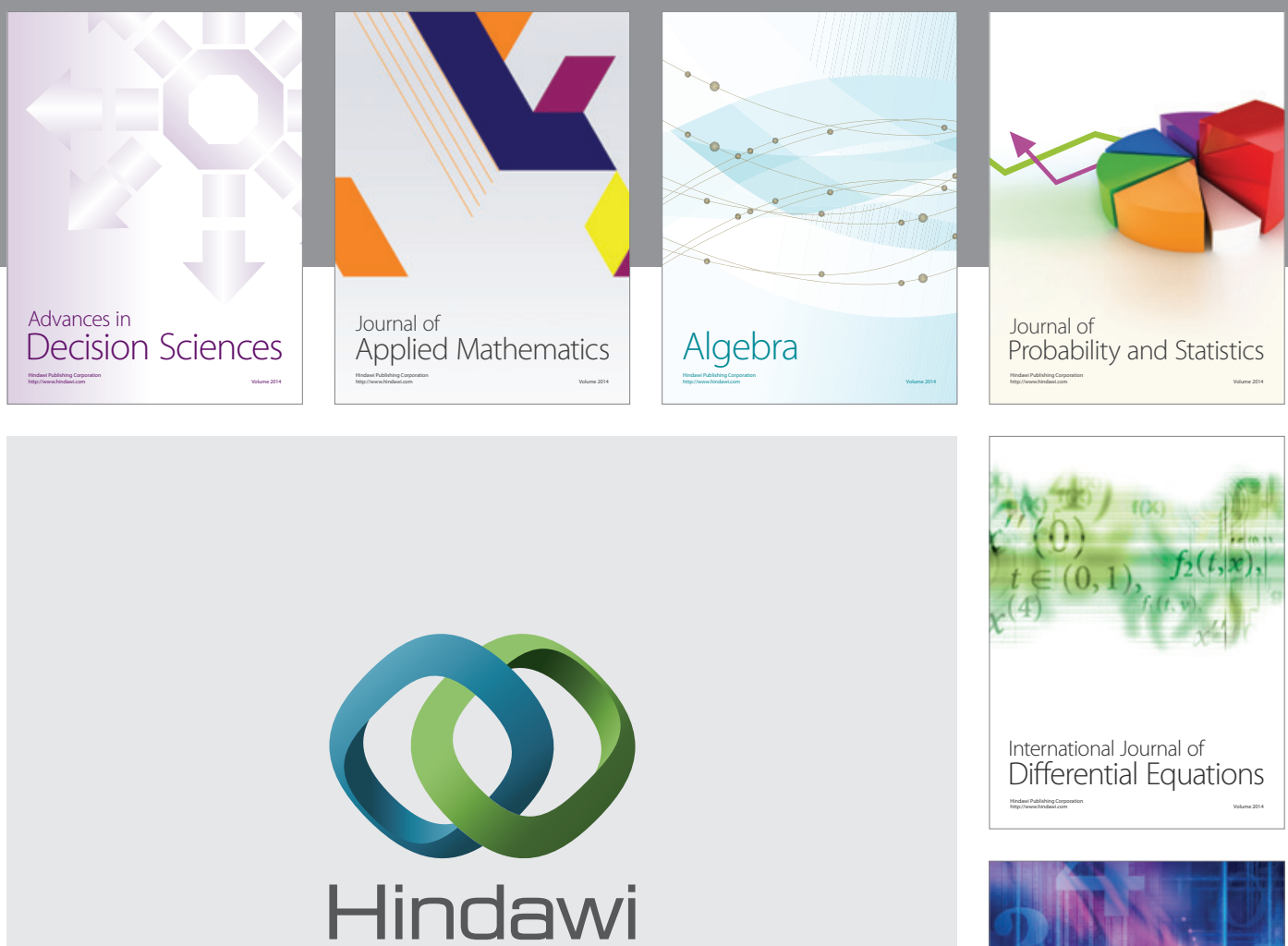

Submit your manuscripts at http://www.hindawi.com
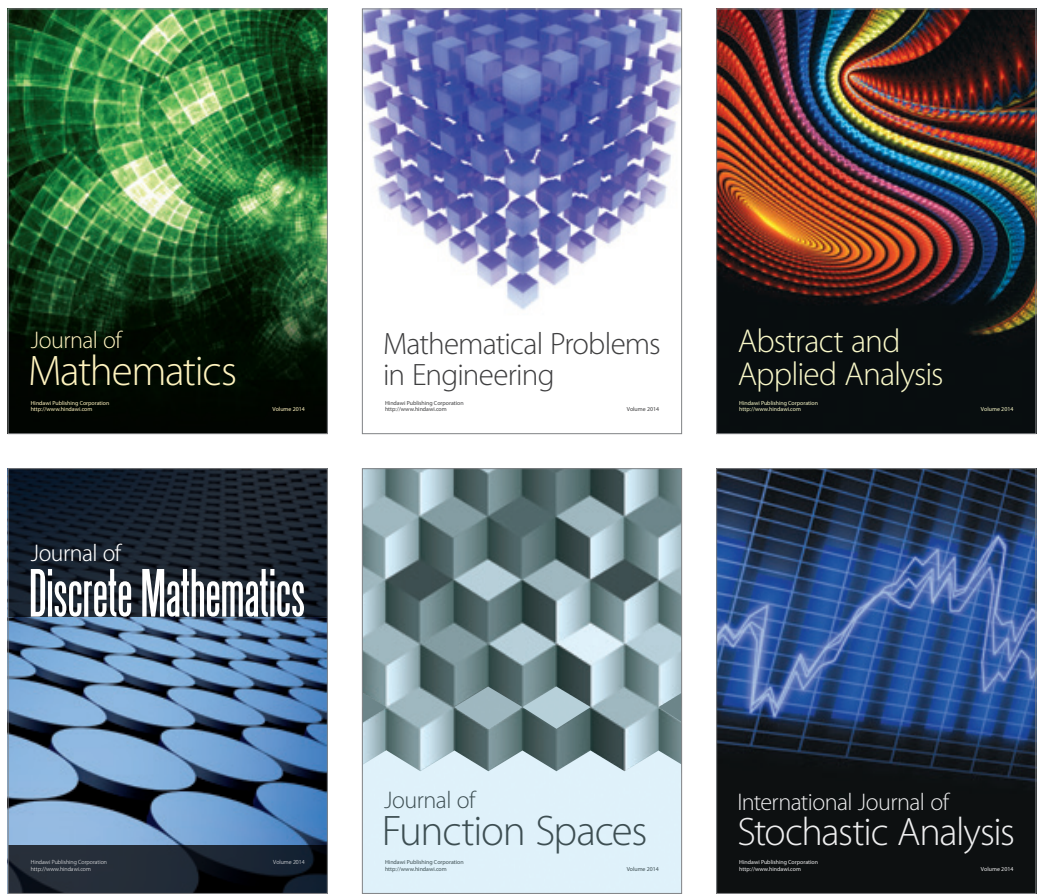

Journal of

Function Spaces

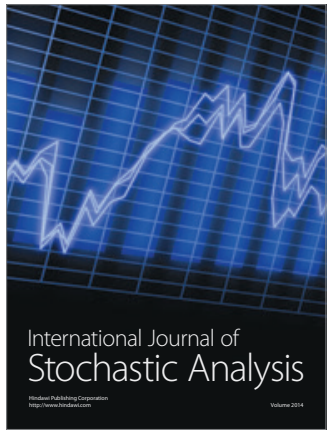

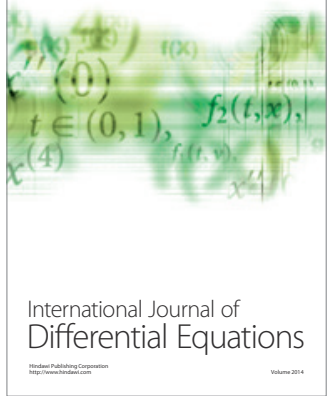
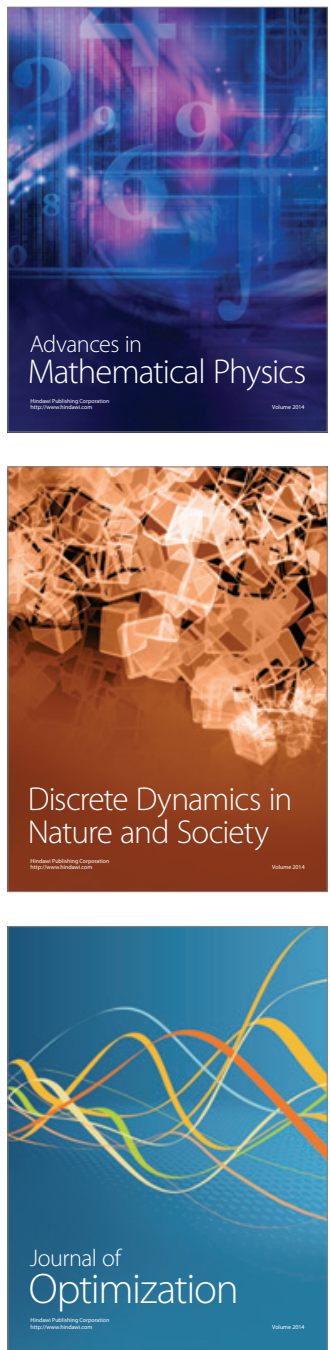